\title{
Impact of botulinum toxin type A on disability and carer burden due to arm spasticity after stroke: a randomised double blind placebo controlled trial
}

\author{
Bipin B Bhakta, J Alastair Cozens, M Anne Chamberlain, John M Bamford
}

\begin{abstract}
Objectives-After stroke, abnormal arm posture due to spasticity in a functionally useless arm may interfere with self care tasks. In these patients botulinum toxin treatment presents an opportunity to reduce disability. The purpose was to investigate whether reduction in spasticity after botulinum toxin treatment translates into reduction in disability and carer burden.

Methods-Forty patients with stroke with spasticity in a functionally useless arm (median duration 3.1 years) were randomised to receive intramuscular botulinum toxin type A (BT-A; Dysport) $(n=20)$ or placebo $(n=20)$ in a total dose of 1000 MU divided between elbow, wrist, and finger flexors. Spasticity (using the modified Ashworth scale), muscle power, joint movement, and pain were assessed. Disability and carer burden were measured using an eight item and a four item scale respectively. Two baseline and three posttreatment assessments (weeks 2, 6, and 12) were made. Concurrent treatments as far as possible remained unchanged and not optimised.
\end{abstract}

Rheumatology and Rehabilitation

Research Unit,

University of Leeds, 36

Clarendon Road,

Leeds LS2 9NZ, UK

B B Bhakta

M A Chamberlain

Department of Rehabilitation Medicine, Woodend Hospital, Aberdeen, UK

J A Cozens

Department of Neurology and Cerebrovascular Medicine, St James

University Hospital,

Leeds, UK

J M Bamford

Correspondence to: Dr B B Bhakta

B.Bhakta@leeds.ac.uk

Received 13 December 1999 and in revised form

6 March 2000

Accepted 23 March 2000
Results-Disability improved at week 6 with BT-A compared with placebo. This effect, present at week 2, wore off by week 12. Reduction in carer burden was seen at week 6 with BT-A and continued for at least 12 weeks. Forearm flexor spasticity after treatment. Although significant improvement in elbow flexor spasticity was seen at week 2 with BT-A compared with placebo, this effect was not evident at weeks 6 and 12. Arm pain was not improved after BT-A. Grip strength was reduced with BT-A. No serious BT-A related adverse effects were reported.

Conclusion-BT-A is useful for treating patients with stroke who have self care difficulties due to arm spasticity. The decision to treat should also include relief of carer burden. As muscle weakness may occur, its potential impact on functional activities must be assessed before intervention.

(F Neurol Neurosurg Psychiatry 2000;69:217-221)

Keywords: stroke; spasticity; botulinum toxin was reduced with BT-A up to 12 weeks
Stroke is a major cause of death and morbidity with an overall age and sex adjusted incidence for first stroke of 2.0/1000/year. ${ }^{1}$ Arm involvement is common with up to $69 \%$ having weakness on admission to hospital. ${ }^{2}$ Long term recovery of arm movement is often poor in patients presenting with a completely paralysed arm with recovery depending on the extent of improvement in the first 6 to 9 weeks. ${ }^{3}$ In patients with a functionally useless arm, abnormal posture due to spasticity may interfere with self care tasks. ${ }^{4}$ In these patients botulinum toxin treatment presents an opportunity to reduce disability.

Botulinum neurotoxin reduces spasticity by blocking acetylcholine release at the neuromuscular junction. ${ }^{5}$ Seven neurotoxin serotypes have been identified, of which botulinum toxin type A (BT-A) is currently used in clinical practice. Botulinum toxin type A offers the possibility of a targeted treatment for "focal" spasticity in the context of managing specific disabilities. It has advantages over systemic treatments which may weaken unaffected muscles and, compared with local treatments such as phenol nerve blocks, sensation is not affected. Although open ${ }^{6-10}$ and randomised control trials ${ }^{11}{ }^{12}$ have shown that BT-A reduces arm spasticity after stroke, its impact on disability and carer burden has been difficult to quantify. Simpson et $a l^{11}$ suggest that future research should explore potential functional gains. To consider this issue, the primary aim of this study was to investigate the impact of BT-A treatment on disability and carer burden in people with arm spasticity after stroke.

\section{Method}

PATIENTS

Fifty four consecutive patients with stroke with chronic hemiparesis referred to the rehabilitation medicine unit for consideration of botulinum toxin for arm spasticity were initially assessed. Twelve patients had functionally useful movement in the paretic arm and were therefore excluded. Two patients declined to participate. The remaining 40 patients, who had finger flexor or elbow flexor spasticity $>2$ on the modified Ashworth score (MAS) ${ }^{13}$ and at least moderate difficulty with two out of eight items defining patient disability (described later), were recruited. Patients had not previously received BT-A or phenol nerve block for spasticity and were at least 6 months poststroke. Approval was obtained from the local 
Table 1 Clinical characteristics and trial drug dosage

\begin{tabular}{|c|c|c|c|c|}
\hline & \multicolumn{2}{|c|}{ Botulinum toxin group $(n=20)$} & \multicolumn{2}{|c|}{ Placebo group $(n=20)$} \\
\hline Female sex & $7(35 \%)$ & & $10(50 \%)$ & \\
\hline Age at stroke ${ }^{\star}$ & $60.2(22.6-77.6)$ & & $53.8(11.2-72.8)$ & \\
\hline Time to recruitment $(\mathrm{y})^{\star}$ & $3.1(0.8-33.2)$ & & $2.7(0.5-15.0)$ & \\
\hline Type of stroke - infarct & $15(75 \%)$ & & $15(75 \%)$ & \\
\hline Haemorrhage & $5(25 \%)$ & & $5(25 \%)$ & \\
\hline Right handed & $18(90 \%)$ & & $20(100 \%)$ & \\
\hline Right hemiparesis & $8(40 \%)$ & & $6(30 \%)$ & \\
\hline \multirow[t]{2}{*}{ Barthel index ${ }^{\star}$} & $13.5(4-20)$ & & $13.5(5-20)$ & \\
\hline & $\begin{array}{l}\text { Botulinum toxin } \\
\operatorname{dose}(M U)\end{array}$ & $\begin{array}{l}\text { Patients } \\
\text { injected }\end{array}$ & $\begin{array}{l}\text { Saline volume } \\
\text { equivalent to dose } \\
\text { in } M U\end{array}$ & $\begin{array}{l}\text { Patients } \\
\text { injected }\end{array}$ \\
\hline \multicolumn{5}{|l|}{ Muscles injected:* } \\
\hline Biceps brachii & $300(100-400)$ & 19 & $300(150-500)$ & 19 \\
\hline Brachioradialis & $100(100-200)$ & 14 & $100(100-200)$ & 12 \\
\hline Flexor digitorum superficialis & $300(200-500)$ & 20 & $300(200-450)$ & 20 \\
\hline Flexor digitorum profundus & $200(100-300)$ & 20 & $200(100-400)$ & 20 \\
\hline Flexor carpi ulnaris & $100(100-200)$ & 13 & $100(100-200)$ & 13 \\
\hline
\end{tabular}

${ }^{\star}$ Median (range).

ethics committee. Written informed consent was obtained from all patients.

\section{STUDY DESIGN}

We performed a single centre, randomised, double blind, parallel trial. Patients received either 1000 mouse units (MU) of BT-A (Dysport) or equivalent placebo (supplied by Ipsen Ltd) diluted in $10 \mathrm{ml} 0.9 \%$ saline. In an attempt to emulate clinical practice some flexibility was allowed between patients in the dose of trial drug given to individual muscles. Dose selection for individual muscles was based on clinical judgement of spasticity as measured by the MAS by a single investigator (BBB). Trial drug was administered by a single investigator (BBB). Ordering, labelling, blinding, recording, and dispensing of trial drug vials were tasks independently undertaken by the pharmacy according to an individual randomisation code produced by the University medical statistics department. Placebo and BT-A were identical in appearance and not accompanied by symptoms at injection that would allow them to be distinguished by patient or researcher. Two baseline assessments were made 1 week apart (weeks -1 and 0 ) before injection of trial drug at week 0 into spastic muscles (table 1) using anatomical landmarks. ${ }^{14}$ Assessments were made at 2, 6, and 12 weeks post-treatment with patients and investigator not permitted to see the responses at previous assessments. The patients completed the disability rating scale and pain score and the carer completed the carer burden scale. All other clinical measurements were performed by a single investigator (BBB). As far as possible existing medical and physical antispasticity treatments were unaltered. One patient died of myocardial infarction after randomisation and therefore no posttreatment measurements were possible (placebo group). One patient was unable to attend the week 2 assessment (placebo group), one patient the week 2 and week 6 assessment (placebo group), and two patients declined the week 12 assessment (BT-A group). The trial was unmasked once all patient assessments had been completed.
OUTCOME MEASURES

As global measures (for example, the Barthel index ${ }^{15}$ ) fail to identify benefits reported by patients, ${ }^{7}$ disability and carer burden scales developed to measure the impact of upper limb spasticity ${ }^{16}$ were used as primary outcomes. The disability scale consisted of eight items (cleaning the palm, cutting fingernails, putting the paretic arm through sleeves, cleaning under the armpit, cleaning around the elbow, standing balance, walking balance, ability to perform a home upper limb physiotherapy exercise programme) and the carer burden scale consisted of four items (cleaning the palm, cutting the fingernails, dressing, cleaning under the armpit). Each item was rated by the patient or carer on a five point Likert scale ("no difficulty" to "cannot do task") at its most difficult over the preceding week. Item scores were summated and divided by the number of items answered giving a summary disability score $(0=$ no disability, $4=$ maximum disability) and carer burden score $(0=$ no carer burden, $4=$ maximum carer burden). The patient completed the rating of disability and the carer completed the carer burden scale. Secondary outcome measures were: (1) Medical Research Council (MRC) muscle power grading ${ }^{17}$ of shoulder abductors, elbow flexors, and extensors and wrist dorsi/palmarflexors; (2) maximum voluntary grip strength (MVG) (Newtons) using hand dynamometry ${ }^{18}$; (3) spasticity in the shoulder adductors, elbow flexor, and finger flexor muscles using the MAS; (4) protractor goniometry for shoulder, elbow, and wrist range of movement (ROM) (5); self completed $0-10$ rating of pain with the limits set by the statements "no pain" and worst pain possible" and scoring based on the worst pain present during the previous week. A separate score for pain in the shoulder, upper arm, elbow, forearm, wrist, and hand was obtained and summated, to obtain a total pain score between 0 and 60 .

\section{STATISTICAL ANALYSIS}

The primary aim was to determine the effect of BT-A on disability and therefore sample size was based on functional change found in a previous study. ${ }^{9}$ Allowing for a placebo response of $30 \%$ and a $90 \%$ chance of detecting a difference between placebo and BT-A at the $5 \%$ significance level, the sample size required in each group is 19. Treatment effect was determined by comparing the change scores between week 0 and week 6 in the BT-A and placebo groups on an intention to treat basis. Week 6 was chosen as the primary end point as any antispasticity effect of botulinum toxin is well established by this time. If a treatment effect was evident then further analysis of data at weeks 2 and 12 was undertaken to gauge the speed of onset and duration of effect respectively. Median and $25 \%$ and $75 \%$ percentiles (IQRs) were used as descriptors. The Wilcoxon matched pairs test was used to analyse ordinal and skewed data. Student's $t$ test was used to analyse MVG. 
Table 2 Baseline variation and changes in disability and carer burden after treatment

\begin{tabular}{|c|c|c|c|c|c|c|}
\hline & \multicolumn{3}{|l|}{ Patient disability* } & \multicolumn{3}{|l|}{ Carer burden * } \\
\hline & $B T-A$ & Placebo & $p$ Value & $B T-A$ & placebo & $p$ Value \\
\hline \multicolumn{7}{|l|}{ Baseline: } \\
\hline Week -1 & $2.0(1.4,2.6)$ & $2.5(2.0,3.0)$ & - & $1.5(0,2.2) \ddagger$ & $1.00(0,1.5)$ & - \\
\hline Week 0 & $2.3(1.5,2.6) \dagger$ & $2.7(2.2,3.1) \dagger$ & - & $2.0(0.2,2.5) \ddagger$ & $1.3(0,1.8)$ & - \\
\hline \multicolumn{7}{|c|}{ Post-treatment change: } \\
\hline Week 0 to 2 & $-0.6(-1.4,-0.3)$ & $-0.1(-0.5,0.2)$ & 0.004 & $-0.77(-1.8,0)$ & $0(-0.4,0)$ & 0.011 \\
\hline Week 0 to 6 & $-0.5(-1.0,-0.2)$ & $-0.1(-0.5,0.1)$ & 0.016 & $-1.0(-1.9,0)$ & $0(-0.3,0)$ & 0.005 \\
\hline Week 0 to 12 & $-0.5(-1.3,0)$ & $-0.2(-0.4,0.1)$ & 0.055 & $-1.0(-2.0,0)$ & $0(-0.3,0)$ & 0.027 \\
\hline
\end{tabular}

${ }^{\star}$ Median (25, 75 percentiles).

†Baseline disability rating significantly greater in placebo group than BT-A group.

¥Variable carer burden rating at baseline.

\section{Results}

Table 1 shows the clinical features of the treatment groups. The four patients with dysphasia had sufficient language ability to give informed consent and complete the assessment protocol. Data on all 20 patients randomised to receive botulinum toxin and 18 out of 20 patients randomised to receive placebo were available for analysis at the primary end point (week 6). Data were not available for two patients in the placebo group, who were unable to attend the week 6 assessment.

\section{PATIENT DISABILITY}

Baseline variation and change in patient disability rating after treatment is shown in table 2. Less disability was reported at week 6 in the BT-A group compared with placebo. A median reduction of 0.5 seems modest in relation to the range of the disability scale (0 to 4$)$. However, the BT-A group had a median pretreatment disability score of 2.3 , with an improvement of $22 \%(0.5 / 2.3)$ at 6 weeks compared with the placebo group, which improved $4.7 \%$. Moreover the BT-A group had less scope to improve as they reported less disability than placebo. Benefits were reported at week 2 but were wearing off by week 12 . Analysis of individual items showed that cleaning the palm, putting the arm through sleeves, doing home physiotherapy exercises, and cleaning the affected armpit were activities most improved with BT-A.

\section{CARER BURDEN}

Thirty six patients out of 40 had carers (BT-A 17 carers; placebo 19 carers). Baseline variation and change in carer burden after treatment is shown in table 2. Physical carer burden was reduced at week 6 with BT-A compared with placebo. Reduction in carer burden was also evident at week 2 in the BT-A group and continued to at least 12 weeks. Reduction in carer burden was particularly evident for the item "cleaning the palm". Eight of 17 patients in the BT-A group who were dependent on carers for this activity became independent whereas all 17 patients in the placebo group who depended on their carers for this activity at baseline remained dependent.

\section{SPASTICITY}

Finger flexor spasticity at baseline was similar between groups (BT-A: median 5, IQR 5, 5; placebo: median 5, IQR 4.3, 5). Finger flexor spasticity improved with BT-A (median change -1.5, IQR-2.8, 0) compared with placebo (median change 0, IQR 0,$0 ; p<0.001$ ) 6 weeks post-treatment (table 3 ). Improvement with BT-A was also present at week 2 and week 12 . Elbow flexor spasticity at baseline was similar between groups (BT-A: median 4, IQR 2, 5; placebo: median 3, IQR 2, 4). Significant reduction in spasticity was evident at week 2 after BT-A compared with placebo; however, no significant difference was evident at weeks 6 and 12 (table 3). There was also no change in shoulder adductor spasticity with BT-A (median change $0, \mathrm{IQR}-1.8,0$ ) compared with placebo (median change $0, \mathrm{IQR}-0.3,0 ; \mathrm{p}=$ $0.22)$.

RANGE OF ARM MOVEMENT

At baseline, only 14/40 had voluntary elbow movement and 3/40 voluntary wrist movement. Active ROM was not changed with BT-A in comparison with placebo. Passive elbow extension was recorded as 0 if full extension was possible (+ve values indicating flexion deformity). There was no improvement in overall passive ROM at the elbow or shoulder between groups. However, in those patients with restriction of passive elbow extension at baseline (BT-A, 7/20; placebo, 4/20) greater improvement was seen with BT-A (median change 11 degrees; range 3-27) compared with placebo (median change 7 degrees; range 0-9) although not statistically significant. Passive

Table 3 Baseline variation and changes in modified Ashworth score after treatment

\begin{tabular}{|c|c|c|c|c|c|c|}
\hline & \multicolumn{3}{|c|}{ Finger flexor spasticity ${ }^{*}$} & \multicolumn{3}{|c|}{ Elbow flexor spasticity ${ }^{\star}$} \\
\hline & $B T-A$ & Placebo & $p$ Value & $B T-A$ & Placebo & $p$ Value \\
\hline \multicolumn{7}{|l|}{ Baseline: } \\
\hline Week -1 & $5(5,5)$ & $5(5,5)$ & - & $3.5(2.6,5.0)$ & $3.0(2.0,4.0)$ & - \\
\hline Week 0 & $5(5,5)$ & $5(4.3,5)$ & - & $4.0(2.0,5.0)$ & $3.0(2.0,4.0)$ & - \\
\hline \multicolumn{7}{|c|}{ Post-treatment change: } \\
\hline Week 0 to 2 & $-2.0(-3.0,-0.3)$ & $0(-0.3,0)$ & $<0.001$ & $-1.0(-1.0,0)$ & $0(-0.25,0)$ & 0.002 \\
\hline Week 0 to 6 & $-1.5(-2.8,0)$ & $0(0,0)$ & $<0.001$ & $-0.5(-1.0,0)$ & $0(-1.0,0)$ & 0.42 \\
\hline Week 0 to 12 & $-1.0(-2.0,0)$ & $0(0,0)$ & 0.006 & $0(-1.0,0)$ & $0(-1.0,0)$ & 0.62 \\
\hline
\end{tabular}

${ }^{\star}$ Median (25, 75 percentiles). 
wrist dorsiflexion improved with BT-A (median change +20 degrees; IQR 5, 31) compared with placebo (median change +3 degrees; IQR-7, 14; $\mathrm{p}=0.006)$. Differences of this magnitude were also seen at weeks 2 and 12 post-treatment.

ARM PAIN

Twenty six $(65 \%)$ of the cohort reported arm pain in the week preceding intervention. All patients were included in the analysis of treatment effect. Baseline pain scores were similar between groups (BT-A: median 6.0, IQR 0, 16; placebo: median 6.0, IQR 0,21). Arm pain was not improved at week 6 (BT-A: median change -2 , IQR -10, 0; placebo: median change 0 , IQR-5.5, 0).

MUSCLE STRENGTH: MRC GRADING

No significant difference in MRC grading was found between the two groups at 6 weeks. MVG was reduced with BT-A (mean change: $-10.7 \mathrm{~N}$ (SD 13.1)) compared with placebo (mean change: $+0.3 \mathrm{~N}$ (SD 10.1)) 6 weeks post-treatment. However the BT-A treated group did have a significantly higher MVG than the placebo group at baseline (BT-A: mean $26.7 \mathrm{~N}$ (SD 18.7); placebo: mean $13.3 \mathrm{~N}$ (SD 15.5)).

\section{ADVERSE EVENTS}

In the BT-A group, two patients developed self limiting arm pain within 1 week of injection and one patient reported worsening of muscle spasm. No serious BT-A related adverse events were reported. In the placebo group, one patient reported herpes labialis 7 days postinjection, one patient reported two transient ischaemic attacks 12 days postinjection, and one patient had exacerbation of cardiac failure 4 weeks after treatment.

\section{Discussion}

This study shows that botulinum toxin can reduce disability and carer burden in people who have long standing stroke with spasticity in a functionally useless arm. This finding is contrary to that reported by Simpson et $a l^{11}$ who did not identify significant effects on disability or carer burden. This may have partly reflected the muscles treated (elbow and wrist flexors only); this would not have affected the flexed finger posture which often causes considerable difficulties with palmar hygiene as stated by the authors. In addition, as this was a dose ranging study comparing three doses of BT-A (BO$\mathrm{TOX}^{\circledR}$ ) and placebo with each group having less than 11 subjects it may not have been powerful enough (although sample size calculations are not given) to detect changes in disability. Our study design also included two pretreatment assessments to document the baseline variability of measurements. Given that improvements after BT-A were greater than the pretreatment variation in self rating of disability and carer burden we can more confidently attribute improvements in these outcomes to BT-A compared with placebo. In our study, although concurrent treatments such as physiotherapy remained unaltered as far as possible they were not optimised, which may account for the modest effect of BT-A on disability, which wore off by week 12 . In addition, BT-A was given to some patients many years after stroke, in whom contractures may have limited its effectiveness.

Our study confirms the improvement in finger flexor spasticity after BT-A reported by Simpson et al. ${ }^{11}$ Although significant reduction in elbow flexor tone was seen at week 2 after BT-A, no difference was apparent at week 6 , again consistent with findings reported by Simpson et $a l^{11}$ and contrary to the open studies. Although improvement in elbow flexor spasticity was limited, improvement in self care tasks (putting the arm through the sleeve) which rely on the ability to passively extend the arm were reported in our study. It is possible that reduction in resistance to passive movement sufficient to ease dressing difficulty may have occurred after BT-A but not enough to change the MAS grade. The use of MAS as a measure of spasticity has recognised limitations ${ }^{20}$ despite being the most widely used outcome measure in spasticity studies.

The only other controlled study of BT-A treatment in patients with stroke with arm spasticity ${ }^{12}$ focused on the impact of electrical stimulation of injected arm muscles on improving the antispasticity effect of BT-A (an effect previously demonstrated by the same author in calf spasticity treatment ${ }^{19}$ ). This study ${ }^{12}$ compared placebo only, placebo+electrical stimulation, BT-A $\left(\right.$ Dysport $\left.^{\circledR}\right)$ only, and BT$A\left(\right.$ Dysport $\left.^{\circledR}\right)+$ electrical stimulation. There were six patients in each group. Although electrical stimulation was reported to increase the effect of BT-A, there were some inconsistencies in the reported antispastic effects of BT-A (as stated by the authors) in that no across group differences were seen in finger flexor spasticity and that $1000 \mathrm{MU}$ BT-A(Dysport ${ }^{\mathbb{B}}$ ) without electrical stimulation did not produce significant reduction in spasticity. These inconsistencies may have related to the small sample size.

Reduction in muscle power may be expected as BT-A prevents acetylcholine release at the neuromuscular junction. In our study, although reduction in proximal muscle strength after BT-A was not identified using the MRC scale, use of a myometer may have identified weakness in proximal muscles given that hand dynamometry identified grip strength reduction after BT-A. Reduction in grip was not identified in a previous controlled study ${ }^{11}$ although in this study finger flexor muscles had not been treated.

We found no analgesic effect of BT-A over placebo contrary to previous open studies. ${ }^{89}$ This may reflect the unselected nature of the patients for pain aetiology. It might be anticipated that pain arising from muscle spasticity or abnormal limb position is more likely to respond to BT-A than pain related to shoulder joint subluxation or "poststroke pain syndrome".

In summary we think that BT-A is useful for treating patients with stroke who have difficulties in self care due to arm spasticity. The decision to treat should not only be based on 
patient disability attributable to spasticity but also on the prospect of relieving carer burden. Although this treatment is easy to administer and well tolerated, muscle weakness may occur and therefore the potential impact of weakness on functional activities must be assessed before intervention. Concomitant use of splints, arm mobilisation exercises, and electrical stimulation $^{12}$ may increase the magnitude and duration of the functional effect of BT-A as well as the possibility of reducing contracture, particularly if used during the early phase of rehabilitation. These assertions need to be explored.

We are grateful to Northern and Yorkshire Regional Health Authority and Ipsen Ltd for funding this study. We thank the
staff of the Community Rehabilitation Unit, St Mary's Hospital, Leeds for their support and Mr Andy Vail, Medical Statistics Unit, University of Leeds for statistical advice.

1 Bamford JM, Sandercock P, Dennis M, et al. A prospective study of acute cerebrovascular disease in the community: study of acute cerebrovascular disease in the community: the Oxfordshire Community Stroke Project 1981-6: methodology, demography, and incident cases of first ever
stroke. F Neurol Neurosurg Psychiatry 1988;51:1373-80.

2 Nakayama H, Jorgensen HS, Raaschou HO, et al. Recovery of upper extremity function in stroke patients: the Copenhagen stroke study. Arch Phys Med Rehab 1994;75:394-8

3 Gowland C. Management of the hemiplegic upper limb in stroke rehabilitation. In: Branstater ME, Basmajan JV, eds. Stroke rehabilitation. Baltimore: Williams and Williams, 1987:217-45.

4 Mayer NH, Esquenazi A, Childers MK. Common patterns of clinical motor dysfunction. Muscle Nerve 1997; (supp 6):21-35

5 Blasi J, Chapman ER, Link E, et al. Botulinum neurotoxin A selectively cleaves the synaptic protein SNAP-25. Nature 1993;365:160-3.

6 Das TK, Park DM. Effect of treatment with botulinum toxin on spasticity. Postgrad Med $\mathcal{F}$ 1989;65:208-10.
7 Hesse S, Friedrich H, Domasch C, et al. Botulinum toxin therapy for upper limb flexor spasticity: preliminary results. f Rehabil Sci 1992;5:98-101.

8 Dunne JW, Heye N, Dunne SL. Treatment of chronic limb spasticity with botulinum toxin A. I Neurol Neurosurg Psychiatry 1995;58:232-5.

9 Bhakta BB, Cozens JA, Bamford JM, et al. Use of botulinum toxin in stroke patients with severe upper limb spasticity. $\mathcal{F}$ Neurol Neurosurg Psychiatry 1996;61:30-5.

10 Sampio C, Ferreira JJ, Pinto AA, et al. Botulinum toxin type A for the treatment of arm and hand spasticity in stroke patients.Clinical Rehabilitation 1997;11:3-7.

11 Simpson DM, Alexander DN, O'Brien CF, et al. Botulinum toxin type $\mathrm{A}$ in the treatment of upper limb spasticity: a randomised double blind placebo controlled trial. Neurology 1996;46:1306-10.

12 Hesse S, Reiter F, Konrad M, et al. Botulinum toxin type A and short term electrical stimulation in the treatment of upper limb flexor spasticity after stroke: a randomised double blind placebo controlled trial. Clinical Rehabilitation 1998;12:381-8.

13 Bohannon RW, Smith MB. Interrater reliability of a modified Ashworth scale of muscle spasticity. Phys Ther 1987;67:206-7.

14 Perotto AO,ed. Anatomical guide for the electromyographer: the limbs and trunk. 2nd ed. Springfeld, USA: Thomas, 1980.

15 Mahoney FI, Barthel DW. Functional evaluation: the Barthel index. MD Med 7 1965;14:61-5.

16 Bhakta BB, Tennant A, Cozens JA, et al. Application of item response theory to measure the disabling effects of severe upper limb spasticity in stroke and the consequent carer burden. Cerebrovascular Dis 1999;9(suppl 1):124

17 Aids to the examination of the peripheral nervous system. London: Medical Research Council, The Stationery Office, 1976. (Memorandum No 45.)

18 Helliwell P, Howe A, Wright V. Functional assessment of the hand: reproducibility, acceptability and utility of a new syshand: reproducibility, acceptability and utility of a new sys-

19 Hesse S, Jahnke MT, Luecke D, et al. Short term electrical stimulation enhances effect of botulinum toxin in the treatment of lower limb spasticity in hemiparetic patients. Neurosci Lett 1995;201:37-40.

20 Pandyan AD, Johnson GR, Price CIM, et al. A review of the properties and limitations of the Ashworth and modified Ashworth scales as measures of spasticity. Clinical Rehat tation 1999;13:373-83. 